\title{
Alphatherapy, the new impetus to targeted radionuclide therapy?
}

\author{
Jean-François Chatal ${ }^{1} \cdot$ Françoise Kraeber-Bodéré ${ }^{2} \cdot$ Michel Chérel $^{1,2} \cdot$ Ferid Haddad $^{1}$ \\ Published online: 25 April 2018 \\ (C) Springer-Verlag GmbH Germany, part of Springer Nature 2018
}

Over the last decade several radiopharmaceutical agents have shown a real benefit for patients who had limited therapeutic options and have been approved. Several clinical studies have been performed with 90 Y-ibritumomab tiuxetan (Zevalin ${ }^{\circledR}$ ) for treatment of follicular lymphoma. The results of a phase III study in 414 patients have proved to be very effective, with a conversion of partial response to complete or unconfirmed complete response and a progression free survival (PFS) of 36.5 months versus 13.3 months for the control group with a relatively high but easily manageable grade $3-4$ hematological toxicity of $66.6 \%$ for thrombocytopenia and $60.8 \%$ neutropenia [1]. A commercial failure after approval can be explained by several non-medical reasons. Moreover, in a phase III clinical trial using ${ }^{177}$ Lu-DOTATE for patients with advanced and progressive midgut neuroendocrine tumor, the estimated rate of progression-free survival at month 20 was $65.2 \%$ as compared with $10.8 \%$ in the control group treated with long-acting repeatable (LAR) octreotide considered as the standard treatment in this clinical situation. The use of ${ }^{177} \mathrm{Lu}$-Dotatate resulted in a risk of progression or death that was $79 \%$ lower than the risk associated with high-dose octreotide LAR. A quite acceptable toxicity rate was observed with less than $10 \%$ grades 3-4 and no renal toxicity over the trial period [2]. Consequently, the radiopharmaceutical agent was approved in 2018.

A real breakthrough in radionuclide therapy occurred in 2013 with the published results of a phase III clinical trial using radium-223 dichloride (Xofigo®), the first alpha-emitting radionuclide being clinically used in patients with metastatic

This editorial commentary refers to the article https://doi.org/10.1007/ s00259-018-4003-6.

Jean-François Chatal

jean-francois.chatal@univ-nantes.fr

1 Groupement d'Intérêt Public Arronax and University of Nantes, Nantes, France

2 ICO-CHU Nantes-UN-CRCINA, Nantes, France castration resistant prostate cancer. The updated analysis involving 921 patients confirmed a survival gain of 3.6 months with regard to the control group (median 14.9 versus 11.3 months) [3, 4]. However, radium-223 dichloride does not bind directly to tumor cells, but indirectly through binding to newly formed bone stroma in close proximity to bone metastases. Despite this limitation, the approval of this radiopharmaceutical in 2013 was a real breakthrough and paved the way for further clinical development of alphatherapy.

The great interest of alpha-emitting radionuclides for therapy is based on a highly cytotoxic effect of alpha particles due to their high linear energy transfer (LET) and a short path length of less than $100 \mu \mathrm{m}$ as compared to electrons of $\beta$ emitting radionuclides that have a low LET and a path length of a few mm resulting in radiation exposure to normal tissue and less effective tumour cell killing effect (5). Consequently it is anticipated that alphatherapy could be more efficient and less toxic than conventional therapy using $\beta$-emitting radionuclides. But, in this age of evidence-based medicine, this advantage has yet to be documented and confirmed in large randomized clinical trials, even though a number of earlystage clinical studies tend to bear this out.

Four alpha-emitting radionuclides have been used in multiple preclinical studies and in a more limited number of earlystage clinical studies [5]: actinium-225, bismuth-213, astatine211 and bismuth-212, the daughter of $\beta$-emitting lead-212. Each has advantages and drawbacks. Actinium-225 is currently the most promising, due to a half-life of 10 days allowing its distribution far from a centralized production site and its easy labelling chemistry for a variety of molecules. However, its major limitation is the mode of production, which needs expensive and rare high-energy accelerators or cumbersome irradiation of radioactive radium-226 targets with questionable availability. Moreover, chelation of the daughter radionuclides of actinium-225 after an alpha decay event is highly questionable and could lead to unwanted side effects. Bismuth-213, a short-lived daughter radionuclide (45.8 $\mathrm{min}$ ) of actinium-225 is available from a ${ }^{225} \mathrm{Ac} /{ }^{213} \mathrm{Bi}$ generator, but the very short halflife of bismuth-213 limits its use to rapidly accessible tumor targets such as hematological bone marrow malignancies or 
after intracavitary instillation. Astatine-211 is available from mid-energy cyclotrons and its half-life of $7.2 \mathrm{~h}$ is more convenient than that of bismuth-213. However, the chemistry of astatine-211 is rich and studies are ongoing to develop labelling for various molecules and its waste should be taken into consideration. Finally bismuth-212 (half-life $60.55 \mathrm{mn}$ ) is available indirectly in hospital from a radium-224 generator (half-life of 3.6 days) extracted from old stocks of ${ }^{232} \mathrm{Th}$. The idea is to use ${ }^{212} \mathrm{~Pb}$ which decays to ${ }^{212} \mathrm{Bi}$ as an in vivo generator to benefit from its physical half-life of $10.64 \mathrm{~h}$. However, rigorous control of the in vivo generator is required to prevent any escape of bismuth with uncontrolled behaviour.

The main problem to be solved in the next ten years will be the availability of these radionuclides for extensive phase II and III clinical studies including hundreds of patients.

Up to now and apart from non-tumor-specific radium-223, more than 15 pilot or phase I/II clinical studies involving a total of 185 patients have been performed worldwide using the four aforementioned radionuclides [5]. The upshot of all these studies is a limited toxicity except for hematological malignancies after intravenous injection, and encouraging and documented efficacy. Nevertheless, the clinical proof of concept for real efficacy has not as yet been documented using the 4 aforementioned radionuclides before implementation of clinical trials phase II/III, which is expected in the coming ten years.

The choice for the most appropriate clinical situations for efficient alphatherapy should take into consideration the physical characteristics of each radionuclide, especially the halflife and the very short path length. Using short-lived radionuclides such as bismuth-213, astatine-211 and lead-212/ bimuth-212, the accessibility of the radiopharmaceutical agent to its tumor target should be easy and fast to allow a high tumor uptake, and the size of the tumor target should fit the very short path length of alpha particles for a maximum tumor cell killing effect. In the current issue of EJNMMI, Autenrieth et al. report the results of a pilot study on alphaimmunotherapy of carcinoma in situ of the urinary bladder using bismuth-213 in 12 patients unresponsive to BCG therapy [6]. The choice for this clinical situation is quite appropriate for an optimal effect of alphatherapy. Plurifocal superficial carcinoma of the urinary bladder is indeed rapidly accessible to intravesically-administered antibody labeled with bismuth213 resulting in fast tumor accessibility. Furthermore the thickness of superficial tumor limited to the mucosa is less than $2 \mathrm{~mm}$, which fits the short path length of emitted alpha particles even if it exceeds its limit of $0.1 \mathrm{~mm}$. These favorable conditions are illustrated by the quite encouraging efficacy results with three complete responses lasting 15, 30 and 44 months and a fourth after a second treatment. Moreover, no toxicity was observed. Such results are impressive for a preliminary pilot study and should be followed by a phase I clinical trial. Specific tumor uptake can be questionable using an intracavitary route and tumor cell killing effect could be due to unspecific effect after intravesical instillation of bismuth-213, but in this hypothesis irradiation of normal mucosa should have led to inflammation and unwanted side effects, which were not reported in this study.

The intracavitary route appears to be preferable for the short-lived radionuclides but obviously does not exclude the intravenous route, especially in situations of isolated tumor cells or clusters of tumor cells disseminated in the body and especially in bone marrow. Encouraging efficacy has been shown in hematological malignancies and prostate cancer especially using actinium-225 [7, 8]. It is highly likely that alphatherapy will progress in the next decade after phase II and III clinical studies in a variety of indications. But the availability of the four aforementioned radionuclides will have to be guaranteed, particularly for actinium-225 through high energy accelerators and, for astatine-211, through a network of mid-energy cyclotrons allowing acceleration of alpha particles. In these conditions, some approvals after the first one using radium-223 could be expected at the end of the next decade. They will come to complement the beta-emitting radionuclides currently available for clinicians.

Funding This work has been supported in part by grants from the French National Agency for Research called "Investissements d'Avenir" Labex IRON n ${ }^{\circ}$ ANR-11-LABX-0018-01 and Equipex ArronaxPlus n ${ }^{\circ}$ ANR11-EQPX-0004, and by grant from INCa-DGOS-Inserm_12558.

\section{References}

1. Morschhauser F, Radford J, Van Hoof A, et al. Phase III trial of consolidation therapy with yttrium-90-ibritumomab tiuxetan compared with no additional therapy after first remission in advanced follicular lymphoma. J Clin Oncol. 2008;26:5156-64.

2. Strosberg J, El-Haddad G, Wolin E, et al. Phase 3 trial of $177 \mathrm{Lu}-$ Dotatate for midgut neuroendocrine tumors. N Engl J Med. 2017;376:125-35.

3. Parker C, Nilsson S, Heinrich D, et al. Alpha emitter radium-223 and survival in metastatic prostate cancer. N Engl J Med. 2013;369:213-23.

4. Hoskin P, Sartor O, O'Sullivan J, et al. Efficacy and safety of radium223 dichloride in patients with castration-resistant prostate cancer and symptomatic bone metastases, with or without previous docetaxel use: a prespecified subgroup analysis from randomized, double-blind, phase 3 ALSYMPCA trial. Lancet Oncol. 2014;15:1397-406.

5. Makvandi M, Dupis E, Engle JW, et al. Alpha-emitters and targeted alpha therapy in oncology: from basic science to clinical investigations. Target Oncol 2018. https://doi.org/10.1007/s11523-018-0550-9.

6. Autenrieth ME, Seidl C, Bruchertseifer F et al. Treatment of carcinoma in situ of the urinary bladder with an alpha-emitter immunoconjugate targeting the epidermal growth factor receptor: a pilot study. Eur J Nucl Med Mol Imaging 2018.

7. Jurcic JG, Ravandi F, Pagel JM, et al. Phase I trial of $\alpha$-particle therapy with actinium-225 (225Ac)-lintuzumab (anti-CD33) and low-dose cytarabine (LDAC) in older patients with untreated acute myeloid leukemia (AML). J Clin Oncol 2015; 33: suppl; abstr 7050.

8. Kratochwil C, Bruchertseifer F, Giesel FL, et al. 225Ac-PSMA-617 for PSMA-targeted $\alpha$-radiation therapy of metastatic castrationresistant prostate cancer. J Nucl Med. 2016;57:1941-4. 